\title{
A Microcontroller Based Voltage Space Vector Modulator Suitable for Induction Motor Drives
}

\author{
Graça Minas \\ University of Minho \\ Dept. of Industrial Electronics \\ Largo do Paço \\ 4709 Braga Codex, Portugal \\ gminas@dei.uminho.pt
}

\author{
Júlio S. Martins \\ University of Minho \\ Dept. of Industrial Electronics \\ Largo do Paço \\ 4709 Braga Codex, Portugal \\ jmartins@dei.uminho.pt
}

\author{
Carlos Couto \\ University of Minho \\ Dep. of Industrial Electronics \\ Largo do Paço \\ 4709 Braga Codex, Portugal \\ ccouto@dei.uminho.pt
}

\begin{abstract}
Pulse width modulation techniques (PWM), introduced two decades ago, are the most used methods to control the voltage and frequency supplied to electrical AC machines. This paper describes a pulse width modulator based on the voltage space vectors technique that accepts voltage demands in dq coordinates and generates three-phase PWM waveforms to drive a variable frequency voltage-source inverter. A scheme based on a high performance 16-bit standard microcontroller with minimum of additional hardware is used, which allows a flexible and economical solution. The output frequency of the modulator can be varied in a large range and with a good resolution. Experimental data obtained from an induction motor drive will be presented.
\end{abstract}

\section{INTRODUCTION}

The continuous efforts to increase industry productivity and to reduce energy costs in a market more and more demanding in terms of product quality, are some reasons that led, in the last two decades, to a great effort in the development of AC motor drives. The development was made in several areas, such as: microelectronics, allowing the use of software instead of hardware to solve complex control problems for an affordable price; power semiconductors technology, providing powerful, fast and easy to drive switches; control algorithms with, for example, the development of very sophisticated and cheaper modulation techniques. As a result of those efforts, $\mathrm{AC}$ motor drives are today a very interesting alternative to $\mathrm{DC}$ motor based systems.

In order to vary the speed of an AC electrical machine, efficiently and in a wide range, both the supply voltage and frequency must be modified [1]. In the solution that nowadays is accepted as standard, these two parameters are adjusted in the DC-AC conversion stage, by means of a PWM voltage-source inverter (Fig. 1).

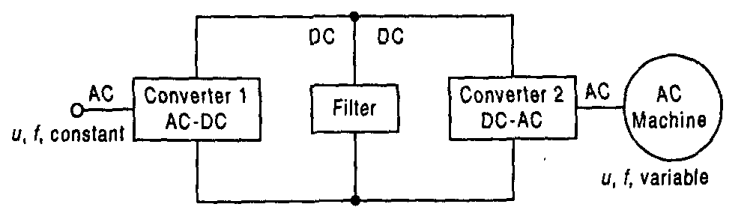

Fig. I. AC drive system.
The main objectives pursued in the PWM modulator described in this paper were:

- to implement a three-phase modulator capable of synthesising waveforms of good quality for a wide range of frequencies and magnitudes and with a good resolution;

- to get a software based solution (the hardware required should be minimum);

- to build a scheme suitable for field oriented control;

- to design the software so that it could be easily integrated in different applications.

\section{DESCRIPTION OF THE MODULATION TECHNIQUE}

The pulse width modulation technique used is based on the voltage space vectors method, which was originally introduced in [2]. The space vectors diagram of line-to-neutral voltages of a voltage-source inverter is shown in Fig. 2. The vectors are presented in the per-unit format where $U_{D C}$ (voltage in the DC bus) is used as base value. They correspond to all the possible states of the inverter output (Fig. 3) and can be designed by integer numbers in the 0 to 7 range. One cycle of the output voltage requires one revolution of the reference vector $\boldsymbol{u}^{*}$ in the $d$-j $q$ complex plane. To synthesise a perfect sine wave, the geometric place of $\boldsymbol{u}^{*}$ should be a circle whose radius corresponds to the desired magnitude. But, when the output voltage is generated by an inverter, only the vectors $\boldsymbol{u}_{0}$ to $\boldsymbol{u}_{7}$ can be produced at a given instant. These vectors are called base vectors. Therefore any vector $\boldsymbol{u}^{*}$ desired must be represented as an average rather than an instantaneous quantity. The average of the base vectors used to produce $u^{*}$ is taken over a switching interval of the inverter, corresponding to the shaded area in Fig. 2 [3]. The reference vector located in a given sector can be synthesised as a linear combination of the two adjacent base vectors $\left(\boldsymbol{u}_{x}\right.$ and $\left.\boldsymbol{u}_{y}\right)$ and one of the two zero vectors $\left(\boldsymbol{u}_{2}\right)$ :

$u^{*}=d_{x} u_{x}+d_{y} u_{y}+d_{z} u_{z}$,

where $d_{x}, d_{y}$ and $d_{z}$ are the duty cycle of the states $X, Y$ and $Z$, respectively, within a switching interval. The duty cycle is defined as the ratio between the duration of the state and the switching interval. So, $d_{x}+d_{y}+d_{z}=1$. 


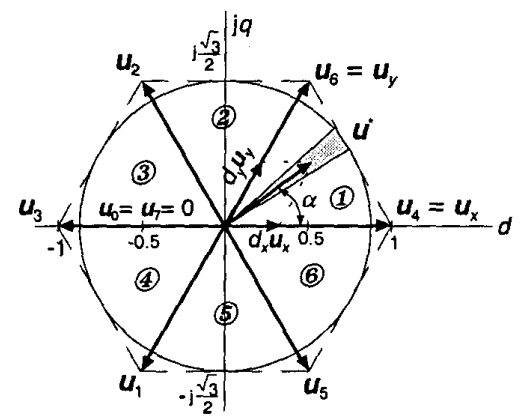

Fig. 2. Voltage space vectors PWM technique.
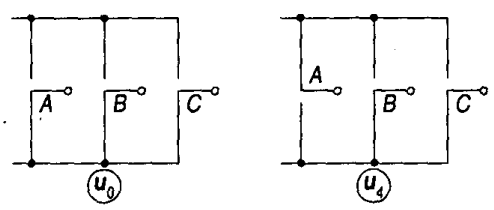

$000_{b}=0_{d}$

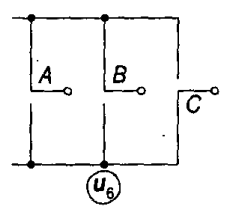

$110_{b}=6_{d}$

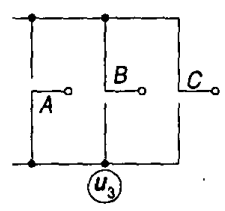

$011_{\mathrm{b}}=3_{\mathrm{d}}$

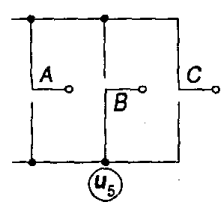

$101_{b}=5_{d}$ $100_{b}=4$
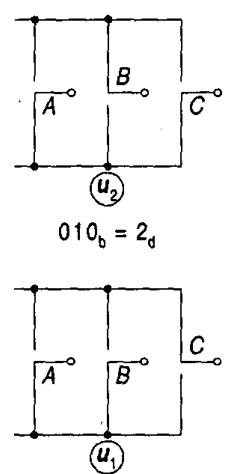

$001_{b}=1_{d}$

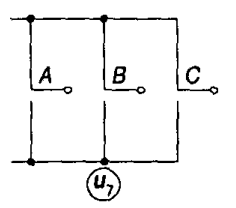

$111_{b}=7_{d}$

Fig. 3. Possible states of an inverter.

In order to avoid low-order harmonics resulting from the non-circular shape of the envelope, the geometrical position of the synthesised vectors is limited to the circle inside the hexagonal envelope (corresponding to the linear modulation in the sinusoidal PWM method). As a result, the maximum magnitude of the reference vector is $(\sqrt{3} / 2) U_{D C}$. To obtain, in the three-phase system, the corresponding line-to-neutral fundamental voltage, this value must be divided by $3 / 2$, giving $U_{L N}=U_{D C} / \sqrt{3}$. This value equals the obtained using the sinusoidal pulse width modulation (SPWM) with harmonic addition, as proved in [4].

The vector $\boldsymbol{u}^{*}$ can also be written, in polar coordinates, as:

$\boldsymbol{u}^{*}=\operatorname{ma} \times U_{\max } \times e^{\mathrm{j} \alpha}$,

where $m a$ is the modulation index $(0<m a \leq 100 \%), U_{\max }$ is the maximum voltage of $\boldsymbol{u}^{*}$ and $\alpha$ its angular position inside the sector. For the example illustrated in Fig. 2, where $\boldsymbol{u}^{*}$ belongs to the sector (1) and $\alpha$ denotes the angular distance between vectors $\boldsymbol{u}^{*}$ and $\boldsymbol{u}_{x}$, we have:

$\boldsymbol{u}_{x}=u_{4}=1+\mathrm{j} 0$

$u_{y}=u_{6}=\frac{1}{2}+\mathrm{j} \frac{\sqrt{3}}{2}$

$\boldsymbol{u}_{z}=u_{0}=u_{7}=0$

After some calculations the duty cycle of the $X, Y$ and $Z$ states can be given by (4). The same rules apply to the other sectors, since the $d$-j $q$ reference frame can be aligned with any base vector.

$d_{x}=\max \sin \left(60^{\circ}-\alpha\right)$

$d_{y}=\operatorname{ma} \times \sin (\alpha)$

$d_{z}=1-d_{x}-d_{y}$

If the $U_{d}$ and $U_{q}$ quantities produced by the controller are written in stator coordinates, as it is usually the case when a field oriented controller is used, $d_{x}$ and $d_{y}$ can be easily computed. In contrast to other methods, there is no need for coordinate transformations or even tables with samples of sinusoidal references. For sector (1), the duty cycle of the states $X$ and $Y$ is:

$d_{x}=\frac{\sqrt{3}}{2} U_{d}-\frac{1}{2} U_{q}$

$d_{y}=U_{q}$

The $Z$ state can be defined by two different base vectors, due to the freedom of choice of the zero vector state, i. e., $\boldsymbol{u}_{0}=(000)$ and $\boldsymbol{u}_{7}=(111)$. In order to assure that the switching from one active state $(X$ or $Y)$ to the $Z$ state involves only the switching of one leg of the inverter, is preferable to choose $\boldsymbol{u}_{0}$ when the previous state is $\boldsymbol{u}_{4}, \boldsymbol{u}_{2}$ or $\boldsymbol{u}_{1}$, and $\boldsymbol{u}_{7}$ will be chosen after $\boldsymbol{u}_{6}, \boldsymbol{u}_{3}$ and $\boldsymbol{u}_{5}$.

If, for example, the vector $\boldsymbol{u}^{*}$ is in sector (1), one possible solution for the pulsewidths for the three-phases is presented in Fig. 4.

For all sectors and in order to keep the sum of $d_{x}, d_{y}$ and $d_{z}$ equal to 1 , the switching sequence and the time of each state $\left(\boldsymbol{u}_{x}, \boldsymbol{u}_{y}\right.$ and $\left.\boldsymbol{u}_{z}\right)$ within a switching interval can be defined as:

$\boldsymbol{u}_{z}\langle d / / 4\rangle \quad \boldsymbol{u}_{x}\left\langle d_{x} / 2\right\rangle \quad \boldsymbol{u}_{y}\left\langle d_{y} / 2\right\rangle \boldsymbol{u}_{z}\langle d / 4\rangle$

$u_{z}\left\langle d_{z} / 4\right\rangle \quad u_{y}\left\langle d_{y} / 2\right\rangle \quad u_{x}\left\langle d_{x} / 2\right\rangle \quad u_{z}\langle d / 4\rangle$,

for the odd sectors, and:

$\begin{array}{lllll}u_{z}\left\langle d_{z} / 4\right\rangle & u_{y}\left\langle d_{z} / 2\right\rangle & u_{x}\left\langle d_{y} / 2\right\rangle & \boldsymbol{u}_{z}\left\langle d_{z} / 4\right\rangle\end{array}$

$u_{z}\left\langle d_{z} / 4\right\rangle \quad u_{x}\left\langle d_{y} / 2\right\rangle \quad u_{y}\left\langle d_{x} / 2\right\rangle \quad u_{z}\left\langle d_{z} / 4\right\rangle$,

for the even sectors. 


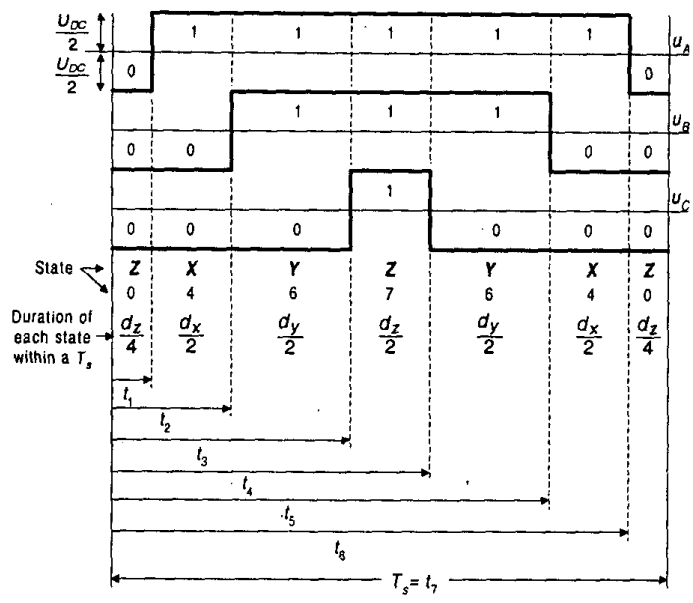

Fig. 4. Synthesis of pulsewidths for the sector (1).

Table 1 summarises the states sequence for one cycle of the output voltage, i. e., one revolution of vector $u^{*}$ in $d$-jq plane (the states sequence are designed by binary numbers which is equivalent to the integer numbers of Fig. 2)

TABLE I

STATE SEQUENCE WITHIN A REFERENCE PERIOD

\begin{tabular}{c|c|c} 
Sector & Position of $\alpha$ & States Sequence (binary) \\
\hline (1) & $0^{\circ} \rightarrow 60^{\circ}$ & $000 \rightarrow 100 \rightarrow 110 \rightarrow 111 \rightarrow 110 \rightarrow 100 \rightarrow 000$ \\
(2) & $60^{\circ} \rightarrow 120^{\circ}$ & $000 \rightarrow 010 \rightarrow 110 \rightarrow 111 \rightarrow 110 \rightarrow 010 \rightarrow 000$ \\
(3) & $120^{\circ} \rightarrow 180^{\circ}$ & $000 \rightarrow 010 \rightarrow 011 \rightarrow 111 \rightarrow 011 \rightarrow 010 \rightarrow 000$ \\
(4) & $180^{\circ} \rightarrow 240^{\circ}$ & $000 \rightarrow 001 \rightarrow 011 \rightarrow 111 \rightarrow 011 \rightarrow 001 \rightarrow 000$ \\
(5) & $240^{\circ} \rightarrow 300^{\circ}$ & $000 \rightarrow 001 \rightarrow 101 \rightarrow 111 \rightarrow 101 \rightarrow 001 \rightarrow 000$ \\
(5) & $300^{\circ} \rightarrow 360^{\circ}$ & $000 \rightarrow 100 \rightarrow 101 \rightarrow 111 \rightarrow 101 \rightarrow 100 \rightarrow 000$
\end{tabular}

\section{DESCRIPTION OF THE MODULATOR}

\section{A. Hardware Design}

A version of the modulator suitable for the field oriented control, accepts as inputs the voltage demand in $d q$ stator coordinates $\left(U_{d}\right.$ and $\left.U_{q}\right)$, and generates, on-line, the three-phase PWM digital waveforms, which drive the power stage (Fig. 5). In the proposed solution, the modulator hardware is just a 16 -bit microcontroller with minimum additional logic, which provides the interface with power stage.

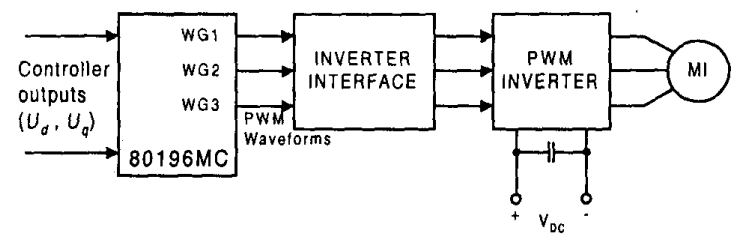

Fig. 5. Block diagram of the used system to test the modulator.

The microcontroller is a standard INTEL MCS-196 microcontroller from the Motion Control family (80C196MC), specially designed for complex, real-time control applications. It shares a common, register-based architecture core that eliminates the accumulator bottleneck and enables fast context switching. Although the MCS-196 microcontrollers are a 16-bit architecture, all devices have bit, byte, word and 32-bit operations. The Motion Control family has peripherals that are optimised for three-phase $\mathrm{AC}$ induction motor control and power inverter applications. These devices have a unique peripheral, the Waveform Generator (WFG), which greatly simplifies the control software and external hardware used for generating three-phase pulse width modulation waveforms. The WFG generates three complementary non-overlapping PWM pulses with resolutions of $125 \mathrm{~ns}$ or $250 \mathrm{~ns}$ (with a $16 \mathrm{MHz}$ oscillator). Once initialised, the WFG requires CPU intervention only to change PWM duty cycles. The WFG features programmable switching (or carrier) frequency up to $32 \mathrm{kHz}$, duty cycle and dead time. The dead time generator (included in the WFG peripheral) prevents the complementary outputs from being turned on at the same time, in order to avoid a short circuit in one leg of the power inverter. This peripheral also has two programmable high drive capability outputs for each phase. The outputs have programmable polarity, or may be forced high or low $[5,6]$.

Fig. 6 shows how the WFG produces the PWM waveforms. The WG_RELOAD register determines the switching frequency. The WG_COUNTER register is a 16-bit counter which is clocked every state machine. When the counter is running, it continuously counts up and down between $0001 \mathrm{H}$ and the WG_RELOAD value. When the counter equals the WG_COMP values (there are three WG_COMP values, one for each phase) the outputs are complemented, so, this register set the pulse width. Each time the WG_COUNTER register reaches the WG_RELOAD value, an interrupt is generated (PI_Interrupt). This interrupt is used to change the WG_COMP register values (if needed).

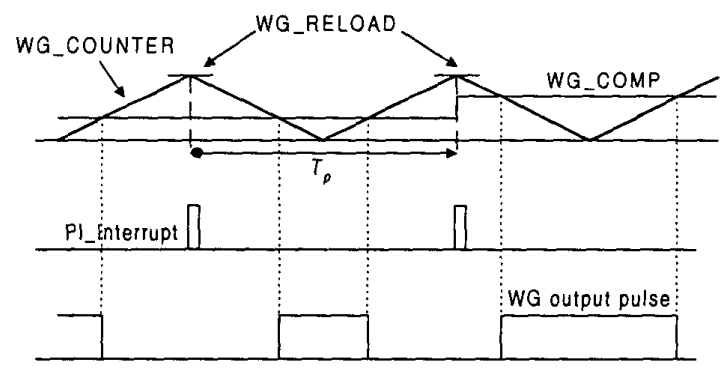

Fig. 6. Carrier and one phase of the output waveform.

\section{B. Software Design}

The general flowchart of the modulator software is shown in Fig. 7. There are basically two concurrent tasks: the CALC_ST and PI_Interrupt routines, Fig. 7 (a) and (b), respectively. In the CALC_ST routine the outputs $U_{d}$ and $U_{q}$ (inputs to the modulator), produced by the controller, are used, firstly, to determine the sector number of the $u$ : vector (using the division of $U_{q}$ by $U_{d}$ ). After that, the duty cycles $d_{x}, d_{y}$ and $d_{x}$ must be computed. Depending on the sector, the equations to compute these duty cycles (5) must be slightly changed, according the sequence given on table 1. Then, the values that must be loaded to the WG_COMP registers are the switching times, $t_{1}, t_{2}$ and $t_{3}$ (Fig. 4), calculated as in (7).

Note that, only the first three switching times must be computed. The WFG produces the remaining switching times $\left(t_{4}\right.$ to $\left.t_{7}\right)$ without CPU overhead. 


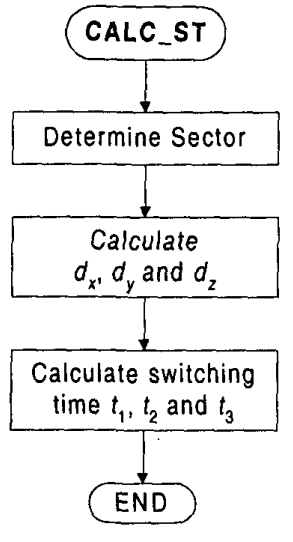

(a)

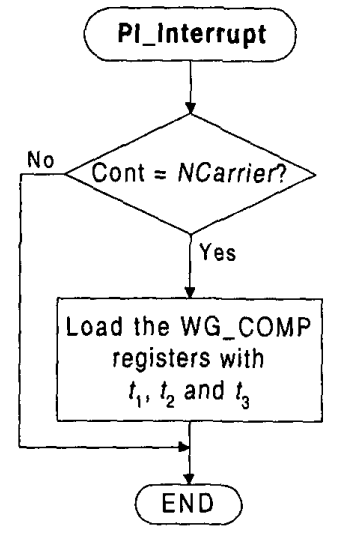

(b)
Fig. 7. Flowchart of the modulator software.

$t_{1}=\frac{d_{2}}{4}$

$t_{2}=t_{1}+\frac{d_{x}}{2}$

$t_{3}=t_{2}+\frac{d_{y}}{2}$

The PI_Interrupt routine is executed within each carrier period (as shown in Fig. 6) and it is the task with higher priority. It just loads the WG_COMP registers with the switching times computed by the CALC_ST routine.

For high carrier frequencies the microcontroller may not be fast enough to compute the switching times for each carrier period. In this case the same switching times are used for more than one carrier period (set by Ncarrier value in the flowchart of Fig. 7(b)).

The minimum pulse width resolution is $250 \mathrm{~ns}$, independently of the WG_RELOAD value. The actual resolution depends on the carrier frequency selected: the higher the carrier frequency, the worst the pulse width resolution.

The carrier frequency used in the experiments was about $16 \mathrm{kHz}$, which allows a pulse width resolution of $1 / 512$, perfectly acceptable for most applications. With this frequency the switching times are updated within four carrier periods. The modulator synthesises a frequency in the range of $0 \mathrm{~Hz}$ to $100 \mathrm{~Hz}$ (with speed inversion) with a resolution of $0.003 \mathrm{~Hz}$.

\section{EXPERIMENTAL RESULTS}

The Fig. 8 presents the hardware arrangement used to test the modulator. A personal computer can be connected to the system through the microcontroller serial port to set the speed reference (input to the controller) and to monitor and adjust some parameters related to the motor operation. The user can choose which parameters he wants to monitor.

The implemented modulator was tested with a $1 \mathrm{CV}$ three-phase induction motor, wye-connected (without neutral), fed by an IGBT voltage-source inverter producing the results presented in Fig. 9 and Fig. 10. The phase-to-middle point of the DC bus (Fig. 9(a) and Fig. 10(a)) and the phase current (Fig. 9(c) and Fig. 10(c)) were acquired on-line. Their corresponding harmonic spectrum (Fig. 9(b), 10(b), 9(c) and 10(c)) were computed with a graphical signal processing tool called Dadisp.

From the figure it can be seen that the voltage spectrum is similar to the one obtained for sinusoidal modulation with triple harmonic addition, in which the most significant harmonic components appear as sidebands centred around the switching frequency and its multiples [7].

With the carrier frequency used, both the current harmonic content and the machine audible noise are very low.

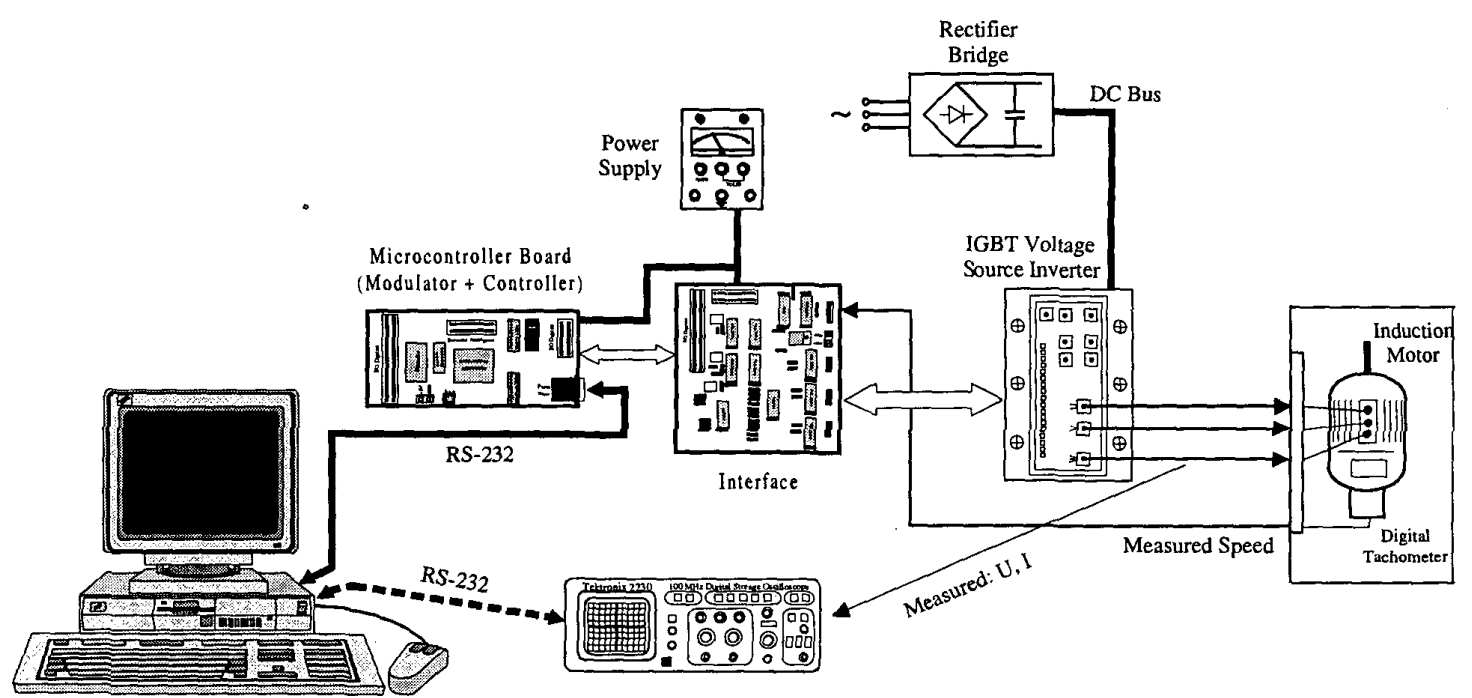

Fig. 8. Hardware arrangement used to test the modulator. 

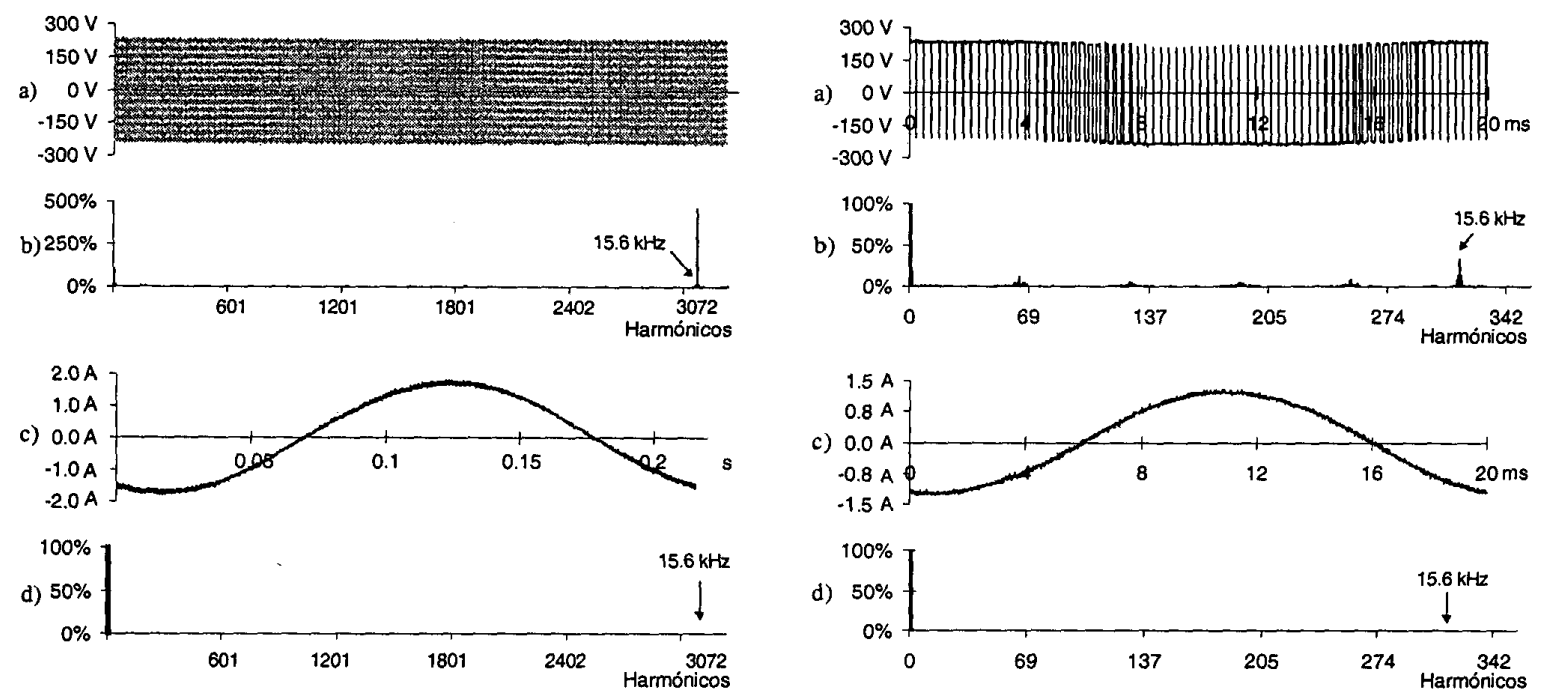

Fig. 9. Voltage space vector modulation $\left(f=5.1 \mathrm{~Hz} ; f_{p}=15.6 \mathrm{kHz}\right)$ : $\begin{array}{lll}\text { (a) voltage waveform; (b) voltage spectrum; } & \text { (c) current }\end{array}$ waveform; (d) current spectrum.

Fig. 10. Voltage space vector modulation $\left(f=50 \mathrm{~Hz} ; f_{p}=15.6 \mathrm{kHz}\right)$ : $\begin{array}{lll}\text { (a) voltage waveform; (b) voltage spectrum; } & \text { (c) current }\end{array}$ waveform; (d) current spectrum.

\section{CONCLUSIONS}

A voltage space vector PWM modulator, suitable for field oriented control, was presented and tested with a $1 \mathrm{CV}$ three-phase induction motor fed by an IGBT voltage-source inverter.

The proposed solution was based on a high performance 16-bit microcontroller with a minimum of additional hardware. The implemented algorithm is very efficient, leaving enough time to implement other tasks with the same microcontroller, like, for instance, simple motor control schemes. High switching frequencies can be achieved with fine resolutions within a large output frequency range.

\section{REFERENCES}

[1] B. K. Bose, ed. lit, Modern power electronics: evolution, technology, and applications. New York: IEEE Press, 1991.

[2] H. W. Broeck, H. Skudelny, G. V. Stanke, "Analysis and realization of a pulsewidth modulator based on voltage space vectors," IEEE Transactions on Industry Applications, vol. 24, $\mathrm{n}^{\circ} 1$, Jan./Fev. 1988, pp. 142-150.

[3] A. M. Trzynadlowski, The field orientation principle in control of induction motors. Boston, Kluwer Academic Publishers, 1994.

[4] G. Minas, Técnicas de modulação de largura de impulso para accionamento de máquinas eléctricas de corrente alternada. Braga, Universidade do Minho, 1998. MSc thesis.

[5] Your guide to Intel's entire line of embedded products and services. Intel Corporation, 1996.

[6] M. Doi, G. Harris, Application examples using the 8XC196MC/MD microcontroller. In "AP-483". Intel Corporation, 1993.

[7] M. H. RASHID, Power electronics: circuits, devices, and appilcations. $2^{\mathrm{s}}$ ed. Englewood Cliffs, NJ: Prentice-Hail, 1993. 